MHSalud, ISSN: 1659-097X, 17(2), Julio-Diciembre, 2020, pp 1-17

Aedo-Muñoz, Abarca-Reyes, Torres-Moreno, Bascuñan-Mosqueira, José Brito, Miarka, Caro-San Juan, Sánchez-Ramírez, Poblete-Gálvez

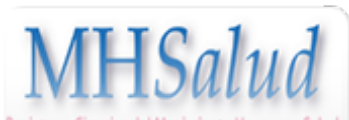

MHSalud

ISSN: 1659-097X

revistamhsalud@una.cr

Universidad Nacional

Costa Rica

\title{
Propuesta de modelo técnico de pateo en fútbol: Una revisión sistemática de variables cinemáticas y cinéticas
}

Aedo-Muñoz, Esteban; Abarca-Reyes, Víctor; Torres-Moreno, María-José; Bascuñan-Mosqueira, Ricardo; José Brito, Ciro; Miarka, Bianca; Caro-San Juan, José; Sánchez-Ramírez, Celso; Poblete-Gálvez, Carolina Propuesta de modelo técnico de pateo en fútbol: Una revisión sistemática de variables cinemáticas y cinéticas MHSalud, vol. 17, núm. 2, 2020

Universidad Nacional, Costa Rica

Disponible en: http://www.redalyc.org/articulo.oa?id=237062748005

DOI: https://doi.org/10.15359/mhs.17-2.6

Esta obra está bajo una Licencia Creative Commons Atribución-NoComercial-SinDerivar 3.0 Internacional. 
MHSALUd, ISSN: 1659-097X, 17(2), Julio-Diciembre, 2020, PP 1-17 Aedo-Muñoz, Abarca-Reyes, Torres-Moreno, Bascuñan-Mosoueira, José Brito, Miarka, Caro-San Juan,

Propuesta de modelo técnico de pateo en fútbol: Una revisión sistemática de variables cinemáticas y cinéticas

Proposal of a Technical Model of Soccer Kicking: A Systematic Review of Kinematic and Kinetic Variables

Proposta de um modelo técnico de pontapé no futebol: Uma revisão sistemática das variáveis cinemáticas e cinéticas

Esteban Aedo-Muñoz

Universidad de Santiago, Chile

esteban.aedo@usach.cl

(iD http://orcid.org/0000-0003-1544-2824

Victor Abarca-Reyes

Universidad de Santiago, Chile

victor.abarca@usach.cl

(D) http://orcid.org/0000-0001-7496-840X

Maria-José Torres-Moreno

Universidad Metropolitana de Ciencias de la Educación,

Chile

mariajose.torresmoreno@hotmail.com

(iD) http://orcid.org/0000-0002-4496-2135

Ricardo Bascuñan-Mosqueira

Universidad Metropolitana de Ciencias de la Educación,

Chile

ricardo.bmosqueira@gmail.com

(D) http://orcid.org/ 0000-0002-5591-5326

Ciro José Brito

Universidad Federal Juiz da Fora, Brasil

cirojbrito@gmail.com

(D) http://orcid.org/0000-0002-9678-1977

Bianca Miarka

Universidad Federal Juiz da Fora, Brasil

miarkasport@hotmail.com

(D) http://orcid.org/0000-0002-7513-7605

José Caro-San Juan

Universidad Metropolitana de Ciencias de la Educación,

Chile

josecarosj@gmail.com

(D) http://orcid.org/0000-0002-7446-9309

Celso Sánchez-Ramirez

Universidad de Santiago, Chile

celso.sanchez@usach.cl

(iD) http://orcid.org/0000-0001-6696-5751

Carolina Poblete-Gálvez
DOI: https://doi.org/10.15359/mhs.17-2.6

Redalyc: http://www.redalyc.org/articulo.oa? id $=237062748005$ 
MHSALUd, ISSN: 1659-097X, 17(2), Julio-Diciembre, 2020, pp 1-17

Aedo-Muñoz, Abarca-Reyes, Torres-Moreno, Bascuñan-Mosqueira, José Brito, Miarka, Caro-San Juan, SÁnchez-Ramírez, Poblete-Gálvez

\author{
Universidad de Santiago, Chile \\ carolina.poblete.g@usach.cl \\ http://orcid.org/0000-0002-4517-9636
}

Recepción: 07 Febrero 2020

Aprobación: 29 Abril 2020

\title{
Resumen:
}

Este artículo presenta una visión propositiva de la bibliografía actual en la técnica de pateo en fútbol. Los objetivos de la investigación son: (i) identificar las variables cinéticas y cinemáticas potenciales utilizadas en los análisis de la técnica y (ii) determinar los valores de variables cinéticas y cinemáticas en relación con la técnica de pateo en fútbol. Se revisaron las siguientes bases de datos; PubMed y Taylor and Francis online, para obtener artículos seleccionados desde 2000-2017. Se utilizó la metodología PRISMA ; se incluyeron artículos con participantes adultos o juveniles sobre la mecánica de pateo en fútbol a través de variables biomecánicas. Los artículos incluidos fueron 29, posteriormente se elaboró un modelo técnico compuesto por cuatro fases: preparación, balanceo, impacto y seguimiento. Una perspectiva biomecánica con base en la técnica podría ofrecer una estrategia prometedora para mejorar, a partir del entrenamiento y análisis de la técnica, el rendimiento en fútbol además de proveer antecedentes para la prevención de lesiones.

Palabras clave: Fútbol, Fenómenos biomecánicos, Cinética, Análisis de desempeño de tareas.

\section{ABstraCt:}

This article describes a propositive vision of the current literature on soccer kicking techniques. It aims to identify the potential kinetic and kinematic variables used in the analysis of the techniques, and to determine the values of kinetic and kinematic variables in relation to the kicking techniques in soccer. PubMed and Taylor \& Francis online were reviewed to obtain selected articles from 2000 to 2017. PRISMA. methodology was implemented. Twenty-nine papers were selected; they considered kicking techniques in soccer young or adult participants through biomechanical variables. Then, a four-phase technical model was prepared: preparation, roll, impact, and follow-up phases. A technique-based biomechanical perspective can offer a promising strategy to improve, from training and technique analysis, the performance in football, in addition to providing a background for injury prevention.

KEYWORDS: Soccer, biomechanical phenomena, Kinetics, Task Performance and Analysis.

\section{Resumo:}

Este artigo apresenta uma visão propositiva da bibliografia atual sobre a técnica do chute no futebol. Os objetivos da pesquisa são: (i) identificar as variáveis cinéticas e cinemáticas potenciais utilizadas na análise da técnica e (ii) determinar os valores das variáveis cinéticas e cinemáticas em relação à técnica de pontapés no futebol. As seguintes bases de dados foram revistas; PubMed e Taylor e Francis online, para artigos selecionados de 2000 a 2017. Foi utilizada a metodologia PRISMA ; foram incluídos artigos com participantes adultos ou juvenis sobre mecânica do chute de futebol através de variáveis biomecânicas. Os artigos incluídos foram 29, posteriormente foi desenvolvido um modelo técnico composto por quatro fases: preparação, balanceamento, impacto e acompanhamento. Uma perspectiva biomecânica baseada na técnica poderia oferecer uma estratégia promissora para melhorar, a partir do treinamento e da análise da técnica, o desempenho no futebol, bem como fornecer um pano de fundo para a prevenção de lesões.

Palavras-Chave: futebol, fenômenos biomecânicos, cinética, análise de desempenho de tarefas.

\section{INTRODUCCIÓN}

El fútbol se caracteriza por tener varias acciones de carácter acíclico durante un partido, éstas se realizan con altas intensidades e incluyen movimientos como: correr, saltar, driblar o patear el balón (Bangsbo, 1994; Bangsbo, et ál., 2006; Mcmillan et ál., 2005). Esta última acción cobra relevancia, ya que, con base en el pateo, se origina el ataque construido, vía por la cual se crean mayor cantidad de ocasiones de gol en los partidos (González-Ródenas, et ál., 2015). Numeros estudios abarcan el pateo en áreas fisiológicas (Stolen, et ál., 2005), psicológicas (Van den Tillaar \& Ulvik, 2014) y, principalmente, en lesiones y sus afecciones (Navandar, et ál., 2018). 
Desde el punto de vista biomecánico, durante la acción del pateo en fútbol se pueden identificar variadas fases, las cuales dependen de su objetivo (Augustus et ál., 2017; Radman et ál., 2016). Éstas deben presentar sus respectivos propósitos mecánicos (PM) (Diener-González \& Aedo-Muñoz, 2017), que hacen referencia a la gran característica mecánica por lograr en casa fase.

En cada PM es necesario obtener los indicadores biomecánicos (IB), también denominados objetivos biomecánicos (OB), correspondientes a todos los indicadores cinemáticos ( $\mathrm{ICm}$ ) e indicadores cinéticos (ICn) derivados del PM. Los ICm son aspectos evaluables por la biomecánica del deporte, enfatizado en el estudio del movimiento, con independencia de las causas que podrían modificarlo; mientras que los ICn corresponden a aquellos que determinan las fuerzas que generan este movimiento (Izquierdo et ál., 2008).

Según Augustus et ál (2017), el pateo en fútbol se engloba en cuatro fases (preparación, balanceo, impacto y seguimiento), las cuales son primordiales para describir la acción mecánica que implican, comenzando desde el tren superior hasta el tren inferior.

Esta revisión tiene por objetivo analizar la bibliografía especializada de variables cinemáticas y cinéticas para generar un modelo técnico actualizado del pateo en fútbol. Estos datos pueden ayudar a profesionales del área de la educación física, ciencias del deporte y afines, para mejorar la calidad de su prescripción del entrenamiento.

\section{Metodología}

El estudio se realizó de acuerdo con la declaración Preferred Reporting Items for Systematic Reviews and Meta-Analyses (PRISMA) (Liberati et ál., 2009; Moher et ál., 2009).

\section{Fuentes de datos}

Para constituir la base de datos del estudio, se examinaron investigaciones y estudios que cumplieran con los criterios de elegibilidad, publicados desde el 1 de enero de 2000 hasta el 31 de diciembre de 2017. Esta selección se realizó en las siguientes bases de datos: PubMed y Taylor and Francis online. Las palabras claves utilizadas de distinto modo y diferentes combinaciones fueron: soccer, biomechanics, kinematic, mechanic, instep kicking, kick. Para las palabras claves se utilizaron palabras booleanas; reducción especifica [AND], ampliación $[\mathrm{OR}]$ y exclusión [NOT].

\section{Criterios de elegibilidad}

Los estudios que cumplían con los criterios de inclusión: (i) varones adultos (27 a 59 años) o juveniles (13 a 26 años) (García-Ruiz et ál., 2010) o varones y mujeres en el mismo estudio; (ii) mecánica de pateo en fútbol a través de variables biomecánicas; (iii) fútbol tradicional; (iv) escritos en inglés y español; y (iv) artículos de investigación primaria.

Dos revisores independientes llevaron a cabo la evaluación y revisión (EA-M y VA-R), y un tercer revisor (M-JT-M) en caso de desacuerdo. Los artículos fueron seleccionados según título y resumen, posteriormente se revisó el texto en extenso y, después se confirmó su inclusión o no en la investigación. 


\section{Recopilación de datos}

Los datos fueron extraídos de todos los artículos considerados apropiados. La información extraída de los artículos correspondió a: característica de la muestra, métodos de medición, variables de mediciones y resultados.

\section{Riesgo de sesgo}

Se realizó una evaluación de la calidad de los estudios incluidos utilizando un formato ajustado de la escala de evaluación de calidad de Newcastle-Ottawa (Wells et ál., 2000). Esta escala contiene ocho ítems categorizados en tres dominios (selección, comparabilidad y exposición), se otorga un máximo de una estrella por cada estudio numerado en los dominios de selección y exposición; mientras que para el dominio de comparabilidad, un máximo de dos estrellas.

\section{Resultados}

\section{Selección de estudios}

La estrategia de búsqueda identificó 485 artículos (Figura 1), de los cuales se eliminaron 14 por estar duplicados. Sus títulos y resúmenes se examinaron para determinar su idoneidad, lo que llevó a la inclusión de 29 artículos de texto completo.

Se excluyeron los estudios que pertenecieran a algunas de las siguientes categorías: estudios publicados en forma de abstract, no escritos en inglés o español, que además no cumplían con la formalidad o rigurosidad para el estudio, con diseño metodológico, intervenciones que utilizaban solo mujeres casos clínicos, revisión de lesiones, programas de prevención de lesiones. 
MHSAlud, ISSN: 1659-097X, 17(2), Julio-Diciembre, 2020, pP 1-17 Aedo-Muñoz, Abarca-Reyes, Torres-Moreno, Bascuñan-Mosqueira, José Brito, Miarka, Caro-San Juan, Sánchez-Ramírez, Poblete-Gálvez

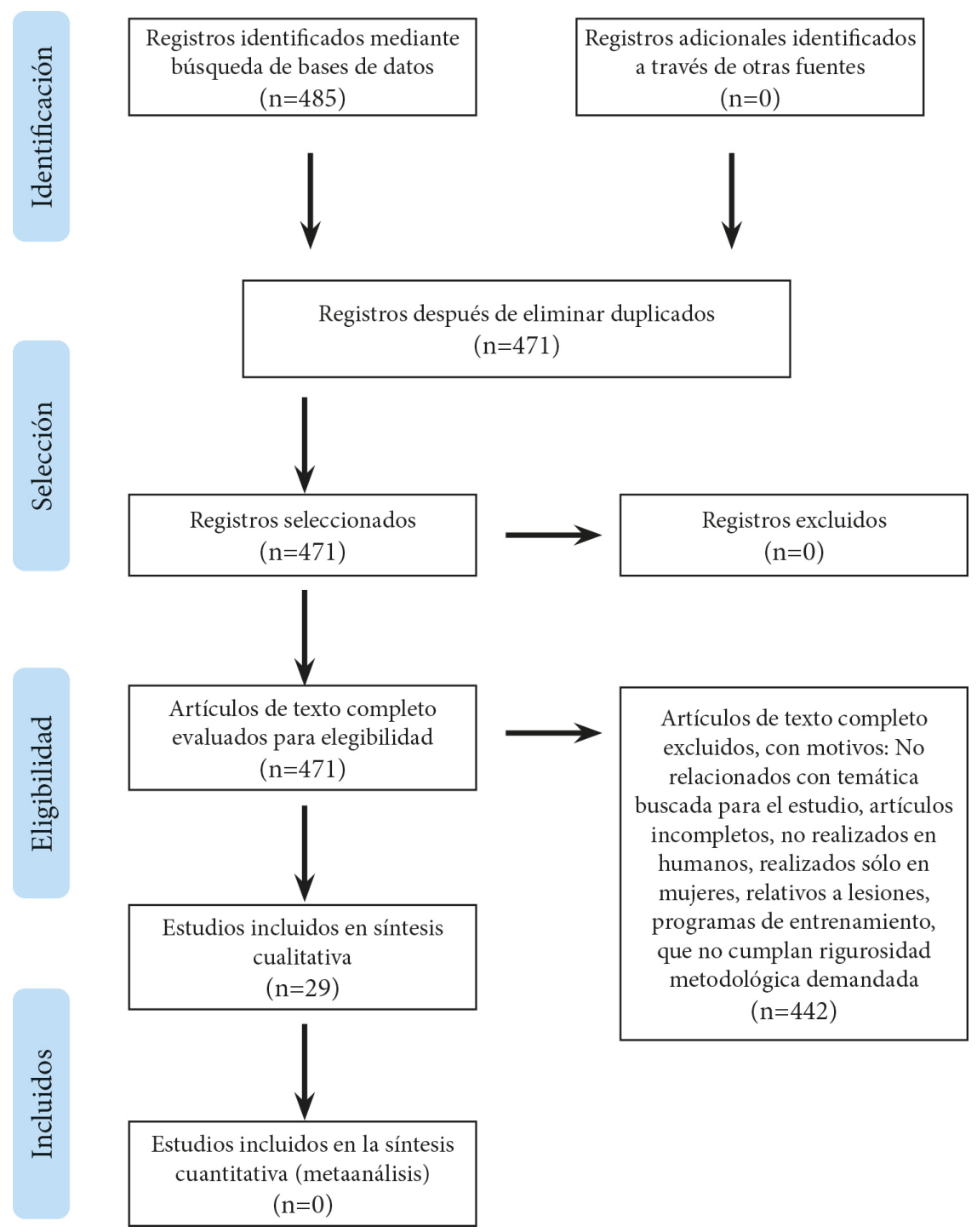

FIGURA 1.

Proceso de selección de artículos PRISMA

Nota: Moher et ál. (2009).

En la Tabla 1(A y B) se detallan los 29 artículos en cuanto a: autor, muestra, método, variables y resultados que se analizaron. 
MHSALUd, ISSN: 1659-097X, 17(2), Julio-Diciembre, 2020, PP 1-17

Aedo-Muñoz, Abarca-Reyes, Torres-Moreno, Bascuñan-Mosqueira, José Brito, Miarka, Caro-San Juan, SÁnchez-Ramírez, Poblete-Gálvez

TABLA 1A.

Descripción de estudios incluidos

\begin{tabular}{|c|c|c|c|c|}
\hline Autor & Muestra & Método & Variables & Resultados \\
\hline $\begin{array}{l}\text { Amiri- } \\
\text { Khorasani et } \\
\text { ál (2016) }\end{array}$ & 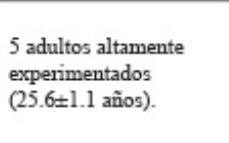 & $\begin{array}{l}10 \text { pateos consecutivos a un objetivo } \\
\text { ubicado a } 3 \text { metros. }\end{array}$ & $\begin{array}{l}\text { Vmáx angular de muslo, } \\
\text { Vmáx angular de la piema, momento } \\
\text { máximo del muslo, Vmáx del balón. }\end{array}$ & $\begin{array}{l}\downarrow \text { velocidad de balón entre la } l^{2} \text { y } \\
\text { la } 5^{2} \text { patada, } \downarrow \text { velocidad angular de } \\
\text { muslo después de la } 6^{2} \text { patada y } \downarrow \\
\text { momento de muslo en } 6^{2} \text { comparado } \\
\text { a la } l^{2} \text { patada. }\end{array}$ \\
\hline $\begin{array}{l}\text { Andersen } \\
\text { T., Dörge H. } \\
(2011)\end{array}$ & $\begin{array}{l}7 \text { experimentados entre } \\
20-33 \text { años, de la } 4^{\mathrm{a}} \text { a } \\
6^{\mathrm{a}} \text { división danesa. }\end{array}$ & $\begin{array}{l}3 \text { pateos para cada condición: I) Con } \\
\text { aproximación y ángulo libres II) } \\
\text { Con demanda de precisión III) Con } \\
\text { acercamiento en línea recta. }\end{array}$ & $\begin{array}{l}\text { Velocidad máxima de carrera, } \\
\text { Velocidad máxima de balón. }\end{array}$ & $\begin{array}{l}\text { La velocidad máxima de balón } \\
\text { ocurre en la } 1^{\star} \text { situación. Cuando } \\
\text { se demanda precisión, la velocidad } \\
\text { disminuye significativamente. }\end{array}$ \\
\hline $\begin{array}{l}\text { Augustus et } \\
\text { ál (2017) }\end{array}$ & $\begin{array}{l}9 \text { experimentados } \\
\text { (23.7 } \pm 3.8 \text { años). }\end{array}$ & $\begin{array}{l}10 \text { pateos máximos con la técnica normal } \\
\text { y } 10 \text { pateos con protocolo de } \mathrm{INT} \text {. }\end{array}$ & $\begin{array}{l}\text { Vmáx de balón, desplazamiento } \\
\text { vertical de cadera. }\end{array}$ & $\begin{array}{l}\text { Después de patear bajo el protocolo } \\
\text { INT, se genera una velocidad de } \\
\text { balón mayor. Lo mismo ocurre con el } \\
\text { desplazamiento vertical de cadera. }\end{array}$ \\
\hline $\begin{array}{l}\text { Charnock et } \\
\text { ál (2009) }\end{array}$ & $\begin{array}{l}15 \text { universitarios de la } \\
\text { liga NCAA }(20.5 \pm 1.4 \\
\text { años). }\end{array}$ & $\begin{array}{l}7 \text { pateos máximos de empeine a una red } \\
\text { ubicada a } 2 \text { metros. }\end{array}$ & $\begin{array}{l}\text { Activación del tren inferior, máx. } \\
\text { extensión de pierna, ratio de } \\
\text { estiramiento de aductor, rotación } \\
\text { externa de cadera que patea, flexión } \\
\text { de rodilla, abducción de cadera (\%). }\end{array}$ & $\begin{array}{l}\uparrow \text { Riesgo de lesión de aductor largo } \\
\text { con el ángulo peak de extensión de } \\
\text { cadera. }\end{array}$ \\
\hline $\begin{array}{l}\text { De Witt et ál } \\
(2012)\end{array}$ & $\begin{array}{l}16 \text { masculinos } \\
\text { experimentados } \\
(24.8 \pm 5.5 \text { años }) \text {. }\end{array}$ & $\begin{array}{l}\text { Un pateo máximo a un balón estacionario } \\
\text { a } 12 \text { metros. }\end{array}$ & $\begin{array}{l}\text { Vmáx de balón, } C M \text { pie, } V \text { angular } \\
\text { peak de pelvis, muslo, piema inferior, } \\
\text { velocidad relativa y peak velocidad } \\
\text { relativa al impactar (rad/s). }\end{array}$ & $\begin{array}{l}\text { Una mayor velocidad del CM del pie } \\
\text { resulta en una mayor velocidad de } \\
\text { balón, al igual que la velocidad máxima } \\
\text { de muslo y velocidad relativa máxima } \\
\text { de rodilla. }\end{array}$ \\
\hline $\begin{array}{l}\text { Fullenkamp } \\
\text { et ál (2015) }\end{array}$ & $\begin{array}{l}10 \text { novatos y } 10 \\
\text { experimentados } \\
(21.8 \pm 2.7 \text { años })\end{array}$ & $\begin{array}{l}5 \text { pateos máximos a modo de } \\
\text { calentamiento, y } 5 \text { pateos máximos con } 1 \\
\text { minuto de pausa entre cada uno. }\end{array}$ & $\begin{array}{l}\text { Vbalón, ROM de rotación de tronco, } \\
\text { peak de velocidad de rotación de } \\
\text { tronco, Vmax de flexión de cadera } \\
(\% / s) \text {. }\end{array}$ & $\begin{array}{l}\text { Correlación } \uparrow \text { entre la velocidad } \\
\text { máxima de rotación de tronco con } \\
\text { la velocidad del balón, así como } \\
\text { la velocidad máxima de flexión de } \\
\text { cadera con la de balón. }\end{array}$ \\
\hline $\begin{array}{l}\text { Inoue et ál } \\
(2014)\end{array}$ & $\begin{array}{l}12 \text { jugadores } \\
\text { expenimentados de } \\
\text { fútbol universitario } \\
(20.9 \pm 0.5 \text { años }) .\end{array}$ & $\begin{array}{l}10 \text { pateos máximos a tres pasos del balón, } \\
\text { a un objetivo central ubicado a } 6 \text { metros. }\end{array}$ & $\begin{array}{l}\text { Vy aceleración angulares de pie, } \\
\text { pierna, muslo, pelvis, Vbalón (N/s). }\end{array}$ & $\begin{array}{l}\leftrightarrow \text { Momentos articulares de tobillo, } \\
\text { pie y rodilla. El movimiento de } \\
\text { extensión de rodilla antes del impacto } \\
\text { al balón contribuiría a la aceleración } \\
\text { del swing de la piema. }\end{array}$ \\
\hline $\begin{array}{l}\text { Ishii et ál } \\
\text { (2009) }\end{array}$ & $\begin{array}{l}5 \text { universitarios } \\
\text { experimentados } \\
(23.4 \pm 0.5 \text { años }) \text {. }\end{array}$ & $\begin{array}{l}\text { Pateos con borde interno a un paso del } \\
\text { balón, a un objetivo ubicado a } 4 \text { metros. }\end{array}$ & $\begin{array}{l}\text { Ángulo de ataque, punto de impacto, } \\
\text { velocidad de balón. Rotación de } \\
\text { balón, Vangular pie inmediatamente } \\
\text { antes de impacto. }\end{array}$ & $\begin{array}{l}\text { El punto de impacto es más influyente } \\
\text { que ángulo de ataque en la velocidad } \\
\text { del balón. La Vbalón se ve afectada } \\
\text { por la Vpie. }\end{array}$ \\
\hline $\begin{array}{l}\text { Jensen et ál } \\
\text { (2014) }\end{array}$ & $\begin{array}{l}10 \text { jóvenes }(15.9 \pm 0.4 \\
\text { años). }\end{array}$ & 45 pateos submáximos y máximos. & $\begin{array}{l}\text { Torque de abducción y aducción de } \\
\text { cadera, torque de flexión de cadera. }\end{array}$ & $\begin{array}{l}20 \text { min de pateos de distinto tipo, } \uparrow \text { el } \\
\text { torque isométrico de cadera después } \\
\text { de } 24 \text { horas. }\end{array}$ \\
\hline $\begin{array}{l}\text { Katis A., } \\
\text { Kellis E. } \\
(2010)\end{array}$ & $\begin{array}{l}10 \text { púberes } \\
\text { experimentados } \\
\text { (13.6 } 0.7 \text { años). }\end{array}$ & $\begin{array}{l}5 \text { pateos máximos con empeine, y } 5 \text { con } \\
\text { borde externo a una portería ubicada a } 7 \\
\text { metros. }\end{array}$ & $\begin{array}{l}\text { V de balón, V de balón/velocidad } \\
\text { pie, Vlineal máxima de tobillo, pie, } \\
\text { cadera, fase de soporte /fase de pre- } \\
\text { soporte. }\end{array}$ & $\begin{array}{l}\uparrow \text { Vbalón en el pateo con empeine. } \\
\uparrow \text { mayor rotación de rodilla/cadera } \\
\text { durante el pateo con el borde extemo. }\end{array}$ \\
\hline $\begin{array}{l}\text { Katis A., } \\
\text { Kellis E. } \\
\text { (2011) }\end{array}$ & 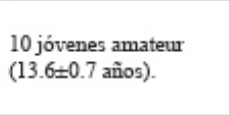 & $\begin{array}{l}\text { Patear } 5 \text { veces al arco haciendo una } \\
\text { maniobra de recorte, y } 5 \text { veces corriendo } \\
\text { directo al balón. }\end{array}$ & $\begin{array}{l}\text { V de balón, relación de Vpie, Vlineales } \\
\text { y angulares en el impacto, Vlineales y } \\
\text { Vangulares máximas, FRS máximos y } \\
\text { en impacto. }\end{array}$ & $\begin{array}{l}\downarrow \text { velocidad del pie y balón en pateos } \\
\text { de empeine después de una maniobra } \\
\text { de recorte reduce la velocidad del pie. }\end{array}$ \\
\hline
\end{tabular}


MHSAlud, ISSN: 1659-097X, 17(2), Julio-Diciembre, 2020, pP 1-17 Aedo-Muñoz, Abarca-Reyes, Torres-Moreno, Bascuñan-Mosoueira, José Brito, Miarka, Caro-San Juan, Sánchez-Ramírez, Poblete-Gálvez

TABLA 2B

Continuación

Continuación...

\begin{tabular}{|c|c|c|c|c|}
\hline Autor & Muestra & Método & Variables & Resultados \\
\hline $\begin{array}{l}\text { Katis et ál } \\
(2013)\end{array}$ & $\begin{array}{l}21 \text { amateur } \\
\text { masculinos }(23.7 \pm 2.3 \\
\text { años). }\end{array}$ & $\begin{array}{l}10 \text { pateos máximos a un objetivo } \\
\text { superior y } 10 \text { a uno inferior. }\end{array}$ & $\begin{array}{l}\text { FRS vertical, anteroposterior, } \\
\text { medio-lateral, EMGs tibial } \\
\text { anterior, recto femoral, biceps } \\
\text { femoral y gastrocnemio. }\end{array}$ & $\begin{array}{l}\uparrow \text { activación electromiográfica } \\
\text { en tibial anterior y recto femoral } \\
\text { durante patadas precisas versus } \\
\text { inexactas. }\end{array}$ \\
\hline $\begin{array}{l}\text { Katis et ál } \\
(2015)\end{array}$ & $\begin{array}{l}10 \text { adultos ( } 25.3 \pm 4.9 \\
\text { años), } 10 \text { adultas } \\
\text { ( } 24.9 \pm 3.5 \text { años) y } \\
10 \text { púberes hombres } \\
\text { (15.1 } \pm 0.7 \text { años). }\end{array}$ & $\begin{array}{l}5 \text { pateos consecutivos máximos, a un } \\
\text { balón detenido a un objetivo ubicado a } \\
5 \text { metros de distancia. }\end{array}$ & $\begin{array}{l}\text { V de balón, Vmáx linear y angular } \\
\text { de cadera, rodilla, tobillo. }\end{array}$ & $\begin{array}{l}\uparrow \text { peak de velocidad de rodilla y } \\
\text { tobillo, y un cambio en el peak de } \\
\text { velocidad de cadera y tobillo más } \\
\text { cerca del impacto en los hombres. }\end{array}$ \\
\hline $\begin{array}{l}\text { Kawamoto et } \\
\text { ál (2007) }\end{array}$ & $\begin{array}{l}7 \text { experimentados } \\
\text { ( } 24.2 \pm 5.5 \text { años) y } \\
8 \text { sin experiencia } \\
\text { (20.7 } \pm 1.5 \text { años). }\end{array}$ & $\begin{array}{l}5 \text { pateos máximos con borde interno, } \\
\text { con distancia de un paso al balón a una } \\
\text { línea objetivo ubicado a } 2 \text { metros. }\end{array}$ & $\begin{array}{l}\text { V de balón, Vangular de cadera, } \\
\text { rodilla, tobillo. }\end{array}$ & $\begin{array}{l}\uparrow \text { velocidad media de balón } \uparrow \\
\text { momento de flexión de cadera en el } \\
\text { grupo experimentado. }\end{array}$ \\
\hline $\begin{array}{l}\text { Naito et ál } \\
(2010)\end{array}$ & $\begin{array}{l}9 \text { universitarios } \\
\text { (21.6 } 1.9 \text { años). }\end{array}$ & $\begin{array}{l}5 \text { pateos máximos a una distancia de } \\
\text { dos pasos del balón a un objetivo a } 5 \\
\text { metros. }\end{array}$ & $\begin{array}{l}\text { Flexión y extensión máxima de } \\
\text { rodilla, Vang máxima de cadera, } \\
\text { Vmáx de extensión de rodilla de } \\
\text { pierna que patea. }\end{array}$ & $\begin{array}{l}\text { Modelo tridimensional de pateo } \\
\text { desarrollado es más útil. El ajuste } \\
\text { entre Vangular de flexión de } \\
\text { cadera y de flexión de rodilla son } \\
\text { más importantes. }\end{array}$ \\
\hline $\begin{array}{l}\text { Nunome et } \\
\text { ál (2007) }\end{array}$ & $\begin{array}{l}5 \text { experimentados } \\
\text { ( } 16.8 \pm 0.4 \text { años })\end{array}$ & $\begin{array}{l}5 \text { pateos con empeine en pierna } \\
\text { dominante y no dominante, a una } \\
\text { portería ubicada a } 11 \text { metros del balón. }\end{array}$ & $\begin{array}{l}\text { Muslo y pierna inferior: Vang } \\
\text { máx., momento muscular de } \\
\text { rodilla y cadera, máx. momento de } \\
\text { interacción, Vmáx de balón, V final } \\
\text { de pie. }\end{array}$ & $\begin{array}{l}\uparrow \text { Vbalón, Vangular pierna, Vfinal } \\
\text { del pie, cuando se patea con la } \\
\text { piema preferida. }\end{array}$ \\
\hline $\begin{array}{l}\text { Orloff et ál } \\
(2008)\end{array}$ & $\begin{array}{l}11 \text { hombres y } \\
12 \text { mujeres, } \\
\text { universitarios de la } \\
3^{2} \text { división NCAA } \\
\text { (20.2 } \pm 1.2 \text { años). }\end{array}$ & $\begin{array}{l}\text { pateos máximos hasta sentir que hizo } \\
\text { alguno a máxima velocidad. }\end{array}$ & $\begin{array}{l}\text { FRS vertical, anteroposterior, } \\
\text { medio-lateral, posición de pierna } \\
\text { de apoyo; ángulo de rodilla, } \\
\text { distancia horizontal desde talón a } \\
\text { balón, UPC. }\end{array}$ & $\begin{array}{l}\text { Inclinación de tronco varia entre } \\
\text { hombres y mujeres, lo que resulta } \\
\text { en mayores peak de fuerzas } \\
\text { laterales. }\end{array}$ \\
\hline $\begin{array}{l}\text { Radman et ál } \\
\text { (2016) }\end{array}$ & $\begin{array}{l}28 \text { hombres }(22.9 \pm 4.8 \\
\text { años). }\end{array}$ & $\begin{array}{l}10 \text { pateos máximos a dos pasos, a un } \\
\text { objetivo ubicado a } 16,5 \text { metros y test } \\
\text { de Leger. }\end{array}$ & Calidad, V y precisión de pateo. & $\begin{array}{l}\text { La fatiga fisiológica ubicada por } \\
\text { sobre el umbral láctico, provoca } \downarrow \\
\text { velocidad de pateo. }\end{array}$ \\
\hline $\begin{array}{l}\text { Shan, } \\
\text { Westerhoff } \\
(2007)\end{array}$ & $\begin{array}{l}7 \text { experimentados y } 8 \\
\text { sin experiencia ( } 20- \\
26 \text { años). }\end{array}$ & $\begin{array}{l}3 \text { intentos máximos con pateo de } \\
\text { empeine, a una distancia de } 3 \text { pasos } \\
\text { desde el balón. }\end{array}$ & $\begin{array}{l}\text { ROM de hombro, codo, cadera, } \\
\text { rodilla y tobillo. }\end{array}$ & $\begin{array}{l}\text { Experimentados muestran mayores } \\
\text { ángulos en cuanto a ROM en } \\
\text { hombro y cadera. }\end{array}$ \\
\hline $\begin{array}{l}\text { Shinkai et ál } \\
\text { (2009) }\end{array}$ & $\begin{array}{l}11 \text { hombres } \\
\text { experimentados } \\
\text { (21.5 } 2.0 \text { años). }\end{array}$ & $\begin{array}{l}10 \text { pateos máximos con empeine a una } \\
\text { portería ubicada a } 11 \text { metros del balón. }\end{array}$ & $\begin{array}{l}\text { Tiempo de contacto con el balón, } \\
\text { Vpie antes de impacto, V después } \\
\text { de impacto, V inicial de balón, ratio } \\
\text { de velocidad pie-balón, fuerza de } \\
\text { reacción del balón. }\end{array}$ & $\begin{array}{l}\text { Duración efectiva para } \uparrow \text { la } \\
\text { velocidad del balón es } 3 / 4 \text { del } \\
\text { tiempo de contacto. El pie que } \\
\text { patea además de dorsiflexión } \\
\text { realiza abducción y eversión. }\end{array}$ \\
\hline $\begin{array}{l}\text { Sidaway et } \\
\text { ál (2017) }\end{array}$ & $\begin{array}{l}30 \text { universitarios } \\
(21.3 \pm 2.2 \text { años). }\end{array}$ & $\begin{array}{l}20 \text { pateos máximos bajo tres } \\
\text { condiciones de estabilidad (agarre } \\
\text { estable, toque ligero, agarre inestable). }\end{array}$ & V de balón. & $\begin{array}{l}\text { Velocidad aplicada al balón es } \uparrow \text { en } \\
\text { condiciones posturales estables. }\end{array}$ \\
\hline $\begin{array}{l}\text { Sinclair et ál } \\
\text { (2014) }\end{array}$ & $\begin{array}{l}22 \text { hombres }(16.8 \pm 0.7 \\
\text { años) }\end{array}$ & $\begin{array}{l}\text { Pateos máximos con empeine a un } \\
\text { balón detenido a una distancia de } 5 \\
\text { metros. }\end{array}$ & $\begin{array}{l}\text { Ángulo pie de pateo, ROM } \\
\text { durante postura, ángulo peak, } \\
\text { ROM relativo desde pie de pateo } \\
\text { a ángulo peak, Vangular pie de } \\
\text { pateo, Vangular al impactar balón, } \\
\text { Vangular peak. }\end{array}$ & $\begin{array}{l}\text { El único predictor significativo } \\
\text { válido de la velocidad de balón, es } \\
\text { la velocidad angular de extensión } \\
\text { de rodilla. }\end{array}$ \\
\hline $\begin{array}{l}\text { Sterzing T., } \\
\text { Hennig E. } \\
(2008)\end{array}$ & $\begin{array}{l}20 \text { hombres } \\
\text { experimentados } \\
\text { ( } 25.4 \pm 3.3 \text { años). }\end{array}$ & $\begin{array}{l}6 \text { pateos máximos al balón por cada } \\
\text { condición de calzado, siendo seis } \\
\text { diferentes tipos. }\end{array}$ & V de balón, FRS del pie de apoyo. & $\begin{array}{l}\leftrightarrow \text { diferencias en velocidad de } \\
\text { pateo de acuerdo con el tipo de } \\
\text { calzado. }\end{array}$ \\
\hline $\begin{array}{l}\text { Van den } \\
\text { Tillaar } \\
\text { (2014) }\end{array}$ & $\begin{array}{l}10 \text { hombres } \\
\text { experimentados } \\
(22.0 \pm 2.5 \text { años })\end{array}$ & $\begin{array}{l}8 \text { pateos en cada condición: a) máximo } \\
\text { sin precisión, b) intentando dar a } \\
\text { objetivo, c) al centro del objetivo, c) lo } \\
\text { más rápido posible, d) al centro. }\end{array}$ & $\begin{array}{l}\text { Vmáx pateo de balón, precisión de } \\
\text { pateo. }\end{array}$ & $\begin{array}{l}\text { Se comprueba que, en las } \\
\text { condiciones de precisión, la } \\
\text { velocidad del balón es entre } 73 \text { y } \\
75 \% \text { de la velocidad máxima. }\end{array}$ \\
\hline
\end{tabular}

Nota: Elaboración propia.

Nota: $\mathrm{V}=$ Velocidad, Vmáx=Velocidad máxima, INT=Intervención de refinamiento técnico, UPC=Unidad de peso corporal, $\mathrm{ROM}=$ Rango de movimiento, $\mathrm{FRS}=$ Fuerza de reacción del terreno, EMG=Electromiografía, Vo=Patear lo más rápido posible en dirección a portería, $\mathrm{Va}=$ Patear lo más rápido posible en dirección al centro del objeto, $A v=$ Golpear el centro del objetivo lo más rápido posible, $\mathrm{Ao}=\mathrm{Golpear}$ el centro del objetivo, $\mathrm{CM}=\mathrm{Centro}$ de masa. 
MHSALUD, ISSN: 1659-097X, 17(2), JuLio-Diciembre, 2020, PP 1-17

Aedo-Muñoz, Abarca-Reyes, Torres-Moreno, Bascuñan-Mosqueira, José Brito, Miarka, Caro-San Juan, SÁnchez-Ramírez, Poblete-Gálvez

\section{Discusión}

Con base en los análisis de movimiento (Shan \& Westerhoff, 2007), se ha definido el objetivo general de rendimiento de la acción (OGR), que correspondería a: Lanzar el balón con la máxima velocidad y precisión. Se han establecido, en este estudio, categorías de análisis respecto de parámetros cinéticos y cinemáticos en común, los cuales comparten varias investigaciones entre sí (Augustus, Mundy \& Smith, 2017; Radman et ál., 2016), que logran incluir los parámetros encontrados en cada fase, para alcanzar el OGR de manera acorde, según sus $\mathrm{PM}$ y $\mathrm{OB}$, de manera específica.

Fase de preparación

Para mejorar la posición del pie de apoyo, y así conseguir estabilidad para la pierna de soporte, nos encontramos con los siguientes comparaciones y contrastes: Para Kawamoto et ál (2007), la duración de la extensión de la pierna de pateo y la aceleración previa al contacto es menor en futbolistas experimentados (72.1 $\pm 14.3 \mathrm{~ms})$, versus novatos (109.4 $\pm 40.3 \mathrm{~ms})$ : los experimentados requieren menor tiempo para alcanzar rangos máximos de movimiento, con lo cual aceleran los segmentos en menor tiempo. Además, los experimentados presentan mayor ángulo de extensión, abducción y rotación externa de cadera y flexión de rodilla, que los novatos. Para Katis et ál (2015), los adultos muestran valores mayores en ángulos de dorsiflexión de tobillo, que los jóvenes. También Katis (2011) informa que antes de que el pie de apoyo toque piso, existen mayores grados de abducción de cadera, cuando la aproximación es recta, o se ejecuta una maniobra de recorte previa al pateo. Ya, Shan \& Westerhoff (2007) revelaron que, cuando se realiza el paso previo a patear, hay diferencias en cuanto al rango de movimiento de cadera en novatos $\left(60 \pm 5^{\circ}\right)$, comparado con experimentados $\left(93 \pm 7^{\circ}\right)$. También, en el rango de movimiento de codo, del hemicuerpo opuesto al pateo, existe un valor mayor en experimentados $\left(158 \pm 12^{\circ}\right)$ versus novatos $\left(54 \pm 5^{\circ}\right)$.

Los valores reportados podrían indicar una mejor coordinación segmentaria previa a la acción de patear en los jugadores experimentados, lo que podría explicar los mayores valores que producen en cuanto a la velocidad de balón; mientras que, según Orloff et ál (2009), los valores de FRS normalizados son mayores

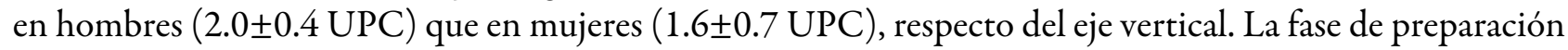
presenta diferencias importantes entre los experimentados y menos experimentados, donde los primeros alcanzan menores tiempos de duración en la fase, mayor desplazamiento angular de las articulaciones, tanto en miembro inferior como en miembro superior.

Fase de balanceo

Para acumular la mayor cantidad de energía que posteriormente pueda transferirse al balón, aumentando la velocidad de la pierna de pateo, nos encontramos con los siguientes comparaciones y contrastes: Katis et ál (2015) informan que la velocidad angular y lineal de segmentos de miembro inferior como cadera, rodilla y tobillo es mayor en hombres adultos, comparado con jóvenes. Fullenkamp et ál (2015) reportan que los experimentados poseen una velocidad angular de rotación de tronco significativamente mayor que los jóvenes ( $428 \pm 117 ; 264 \pm 79^{\circ} \%$ respectivamente). Esto, en parte, podría explicar que el miembro superior contribuye de manera importante al rendimiento del pateo. Augustus et ál (2017) mencionan que, al aplicar un protocolo de intervención para aumentar el rango de movimiento de cadera, el desplazamiento del centro articular de cadera aumenta significativamente $(0.04 \pm 0.01 \mathrm{~m})$ comparado con la condición normal, sin intervención $(0.03 \pm 0.01 \mathrm{~m})$. Además, Fullenkamp et ál (2015) manifiestan que el rango de movimiento de tronco es mayor en experimentados comparado con jóvenes, $40 \pm 10^{\circ}$ y $26 \pm 9^{\circ}$; respectivamente. Katis et ál (2011) comparan un pateo de empeine con aproximación recta, versus un pateo con una maniobra de recorte, y concluyen que durante la maniobra de recorte hay un mayor grado de rotación interna de cadera.

Además, Inoue et ál (2014) detallan valores de velocidades angulares de articulaciones de cadera, rodilla y tobillo de la pierna de apoyo. Durante la fase temprana, desde que la pierna que patea comienza a acelerar previa al impacto ( $25 \%$ fase de balanceo), se observa un movimiento consistente de abducción de tobillo. Por otra parte, observan un ligero movimiento de aducción, mientras la abducción de tobillo 
estaba ocurriendo. Así mismo, durante el $50 \%$ del tiempo desde el contacto del pie de apoyo con el piso, hasta el balón, se registra el mayor valor de velocidad angular del segmento de cadera. De Witt \& Hinrichs (2012) informan valores en velocidades pico angulares de pelvis $(735.7 \pm 281.3 \%$ s $)$, muslo $(758.0 \pm 127.8 \%$ s) y pierna inferior $(1312.6 \pm 119.7 \%$ s), previo al contacto con el balón. Katis et ál (2011) observaron que, durante una aproximación recta al balón, las velocidades angulares de cadera, rodilla y tobillo son mayores respectivamente $(774.4 \pm 79.3 ; 1625.6 \pm 160.8 ; 1765.8 \pm 162.4 \%$ s) en comparación con una maniobra de recorte previa $(707.3 \pm 81.7 ; 1539.5 \pm 194.4 ; 1571.0 \pm 177.6 \%$ s).

Para Nunome et ál (2007), la velocidad máxima angular de pierna se alcanza justo antes del impacto con el balón, pero también muestran que la velocidad angular máxima de muslo se produce alrededor de $75 \%$ del total de la fase comprendida al patear, donde es $100 \%$ el impacto al balón y disminuye a valores iniciales al contacto. Esto, sin duda, explica que el muslo ayuda a la aceleración en una fase temprana, mientras que la extensión de rodilla es el principal contribuyente. Charnock et ál (2009) reportan que, durante los momentos previos a patear, la activación del aductor largo alcanzó su mayor valor cercano al $30 \%$ en la fase previa a tomar contacto con el balón. Además, el máximo cambio de longitud de este músculo sucede entre el 40-60 \% de la fase previa a impactar el balón, en donde la pierna que patea se encuentra acelerando.

Brophy et ál (2007) compararon parámetros en pateo con borde interno y empeine, detallaron que, durante el pateo con borde interno, la activación del tibial anterior, en fases previas al contacto con el balón, en que la pierna de pateo comienza a acelerar, presenta una mayor actividad comparada con el mismo músculo en pateo con empeine. Esto podría indicar que, para esa acción técnica específica, se necesita la mayor activación del tibial anterior para ejecutar correctamente el pateo y que, justamente, es un rasgo distintivo que diferencia al pateo con borde interno, del pateo con empeine. Los valores de la fase de balanceo demuestran que los jugadores experimentados tienen mayores velocidades angulares de las articulaciones como lineales de los distintos segmentos entre los que destacan el muslo y la pierna, el primero alcanza su máxima velocidad antes de impactar el balón, mientras que la pierna lo realiza en el momento de contacto con el balón. Esto explica que las primeras aceleraciones provienen del muslo y se transfieren hacia la pierna y el pie.

Fase de impacto

Para imprimir la mayor cantidad de energía acumulada en la fase anterior, si se dirige el objetivo al lugar escogido por el pateador, es decir, se transfiere esta velocidad al balón, se encuentran las siguientes comparaciones y contrastes: Para el tiempo de duración en un pateo, Brophy et ál (2007) encontraron diferencias en universitarios, en cuanto a la duración del pateo con empeine $(0.79 \mathrm{~s})$, comparado con el pateo con borde interno $(0.83 \mathrm{~s})$. Para Katis \& Kellis (2010), se alcanzan velocidades mayores cuando se patea con empeine $(19.6 \pm 1.9 \mathrm{~m} / \mathrm{s})$ versus borde externo $(18.1 \pm 1.5 \mathrm{~m} / \mathrm{s})$. Andersen y Dörge (2011) muestran que la velocidad de balón es diferente con distintas formas de aproximación, por ejemplo, cuando ejecutaban un pateo con empeine y un ángulo de aproximación autoseleccionado, se registraban velocidades mayores (28.6-34.5 m/s), que cuando la aproximación es recta, $(25.6-32.3 \mathrm{~m} / \mathrm{s})$; mientras, Kawamoto et ál (2007) reportan que la velocidad de balón en experimentados $(21.4 \pm 1.5 \mathrm{~m} / \mathrm{s})$ es mayor que en novatos $(16.0 \pm 1.0$ $\mathrm{m} / \mathrm{s}$ ). Por otro lado, Nunome et ál (2007) analizaron en experimentados el pateo con empeine en pierna dominante versus no dominante y encontraron que la pierna dominante presenta valores más altos en algunos parámetros estudiados, versus la no dominante, con mayores valores para la velocidad del balón (32.1 vs. 27.1 $\mathrm{m} / \mathrm{s})$, velocidad angular de pierna (2257.5 vs. $1822 \%$ s) y velocidad final del pie ( $22.7 \mathrm{vs.} 19.6 \mathrm{~m} / \mathrm{s})$.

De acuerdo con Sidaway et ál (2017), en condiciones estables las velocidades de balón son mayores $(15.9 \pm 2.97 \mathrm{~m} / \mathrm{s})$ que en inestabilidad $(13.7 \pm 2.0 \mathrm{~m} / \mathrm{s})$. Augustus et ál $(2017)$ han reportado que la velocidad del balón es mayor al aplicar un protocolo de intervención de aumento de flexibilidad en extensión de cadera $(26.3 \pm 2.1 \mathrm{~m} / \mathrm{s})$ versus condiciones normales de pateo $(25.1 \pm 1.5 \mathrm{~m} / \mathrm{s})$. Por otro lado, Radman et ál (2016) concluyen que diversos parámetros como velocidad del balón, precisión y calidad disminuyen significativamente en condiciones de fatiga aguda. Katis et ál (2015) registran que la velocidad del balón es mayor en hombres adultos versus jóvenes $(21.5 \pm 2.0 ; 19.4 \pm 1.88 \mathrm{~m} / \mathrm{s})$. Además, se encuentra que los 
MHSALUD, ISSN: 1659-097X, 17(2), JuLIO-DiCIEMBRE, 2020, PP 1-17

Aedo-Muñoz, Abarca-Reyes, Torres-Moreno, Bascuñan-Mosqueira, José Brito, Miarka, Caro-San Juan, SÁnchez-Ramírez, Poblete-Gálvez

valores en la flexoextensión de rodilla son significativamente mayores en adultos, durante la fase final de pateo. Fullenkamp et ál (2015) han comparado futbolistas experimentados versus novatos, y reportan que los primeros muestran mayores velocidades de pateo $(25.6 \pm 2.2$ y $19.5 \pm 2.0 \mathrm{~m} / \mathrm{s}$; respectivamente). Por otro lado, Van den Tillaar, \& A. Ulvik (2014) demuestran que las velocidades que alcanza el balón son significativamente mayores cuando la instrucción dada es patear con fuerza y sin precisión.

Sinclair et ál (2014) presentan diferentes valores en velocidades angulares para la pierna de apoyo y la que patea, tanto en cadera, rodilla y tobillo. Los valores registrados en ese orden, respectivamente, al impactar con el pie son: $45.6 \pm 110.7 \%$ s; $-270.7 \pm 115.3 \%$ s; $544.1 \pm 167.9 \%$ s; $-10.4 \pm 145.0 \%$ s; $-54.1 \pm 183.1 \%$; $-359.3 \pm 176.6 \%$ s. Además, Amiri et ál (2011) evaluaron diez pateos consecutivos, encontrando que a partir del quinto la velocidad angular de muslo y pierna son significativamente inferiores $(1012.4 \pm 10.3$; $2158.3 \pm 23.5 \%$ s), comparado al primer $(1078.9 \pm 16.6 ; 2306.7 \pm 24.6 \%$ s). Inoue et ál (2014) han reportado valores para la velocidad del balón $(26.6 \pm 1.5 \mathrm{~m} / \mathrm{s})$; De Witt \& Hinrichs (2012) reportan valores similares $(26.5 \pm 2.1 \mathrm{~m} / \mathrm{s})$. Katis et ál (2011) comparan las velocidades del balón con acercamiento recto $(19.4 \pm 1.8 \mathrm{~m} /$ $\mathrm{s})$, y resultan mayores que las registradas con una maniobra de recorte $(17.1 \pm 1.5 \mathrm{~m} / \mathrm{s})$. Katis et ál (2013) cuantificaron las FRS al momento del impacto, durante tiros dirigidos hacia la parte superior de la portería, y otros hacia la parte inferior, en los tiros superiores. Sus resultados informan que las FRS son mayores en el eje vertical $(480.5 \pm 255.8 \mathrm{~N})$, anteroposterior $(128.0 \pm 74.2 \mathrm{~N})$ y mediolateral $(359.7 \pm 92.5 \mathrm{~N})$ para los tiros que no daban en el objetivo versus los que sí daban en él (449.4 $\pm 296.4 ; 125.4 \pm 88.2 ; 331.2 \pm 89.5 \mathrm{~N})$. Respecto de

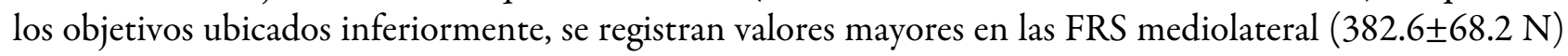
y anteroposterior $(85.6 \pm 78.6 \mathrm{~N})$ en los tiros correctos, comparado con los que no acertaban $(81.0 \pm 50.6 \mathrm{~N}$ anteroposterior, $370.6 \pm 74.5 \mathrm{~N}$ mediolateral).

Respecto de la activación muscular, Katis et ál (2013) reportan datos útiles que se presentan en algunos grupos musculares, donde comparan tiros dirigidos a objetivos superiores e inferiores, además evalúan parámetros de activación cuando el tiro fue correcto o incorrecto. Observan una activación significativamente menor en tibial anterior (22.2 $\pm 14.4 \%$ MVCRMS), bíceps femoral (35.5 \pm 23.4 \%MVCRMS) cuando el tiro fue erróneo, en los tiros dirigidos hacia arriba, comparado con los tiros que

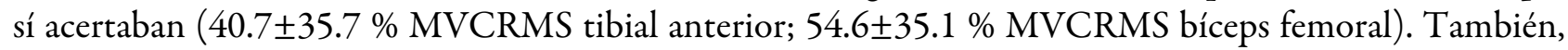

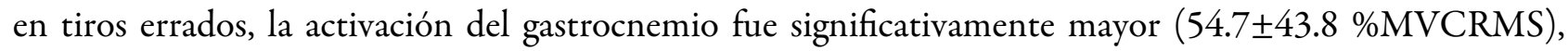
comparada con tiros en los que sí se acierta (37.4 \pm 24.5 MVCRMS). Así mismo, se registran valores significativamente mayores para los tiros errados a objetivos inferiores, en tibial anterior $(31.5 \pm 26.9$ \%MVCRMS) y recto femoral (60.2 $\pm 35.6 \% \mathrm{MVCRMS})$, comparado con la activación muscular en los mismos grupos, para tiros acertados (17.8 $\pm 14.8 \%$ MVCRMS tibial anterior; $40.1 \pm 27.7 \%$ MVCRMS recto femoral). Brophy et ál (2007) plantean que existe una mayor activación del tibial anterior, en distintas fases del pateo con borde interno, en comparación con el pateo con empeine.

Sinclair et ál (2014) muestran, en diferentes articulaciones como cadera, rodilla y tobillo, distintos valores respecto al rango de movimiento y ángulos para la pierna de apoyo y la pierna que patea. Así, los valores durante el impacto de balón para pie de apoyo, y de pateo, en cadera, rodilla y tobillo, respectivamente son: Ángulo de impacto al balón: $11.9 \pm 13.85^{\circ} ; 13.1 \pm 10.31^{\circ} ; 41.6 \pm 10.8^{\circ} ; 42.0 \pm 9.6^{\circ} ; 51.98 \pm 24.9^{\circ} ; 77.5 \pm 11.6^{\circ}$. Rango de movimiento: $27.2 \pm 8.6^{\circ} ; 39.99 \pm 13.9^{\circ} ; 36.4 \pm 19.4^{\circ} ; 18.1 \pm 11.7^{\circ} ; 10.7 \pm 4.2^{\circ} ; 4.3 \pm 3.7^{\circ}$. Ángulo al impactar con el pie: $-24.7 \pm 6.5^{\circ} ; 51.1 \pm 11.4^{\circ} ; 77.8 \pm 15.1^{\circ} ; 24.4 \pm 7.1^{\circ} ; 46.3 \pm 15.7^{\circ} ; 81.7 \pm 10.2^{\circ}$. La fase de impacto es, sin duda, la más relevante para muchos investigadores, entrenadores y profesores, pero gran parte de sus registros se deben al resultado de fases previas. Esta fase podría mejorar cuando las trayectorias de acercamiento del jugador no son necesariamente rectas y el impacto con el balón se realice con la pierna dominante. Estos dos factores aumentan la velocidad de impacto con el balón, mientras que para mantener estos niveles elevados de velocidad en tiros consecutivos se recomienda que el jugador ejecute solo cinco tiros antes de que la velocidad disminuya; a nivel muscular cualquier variación de actividad eletromiográfica del tibial anterior es importante para que la dirección del tiro sea errado. 
Fase de seguimiento

Para estabilizar el cuerpo para una siguiente acción, que reduzca la inercia, nos encontramos con los siguientes comparaciones y contrastes: Amiri-khorasani et ál (2011) detallan que los rangos de movimiento en cadera después de sesiones de estiramientos dinámicos son mayores $\left(24.2 \pm 3.1^{\circ}\right)$ que cuando se realizan estiramientos estáticos $\left(20.7 \pm 7.9^{\circ}\right)$, lo que demuestra que estiramientos dinámicos contribuirían a mejorar el rendimiento durante el pateo. Por otro lado, los valores encontrados por Shinkai et ál (2009) demuestran que, posterior al impacto con el balón, existe una reducción en la eversión del pie, alrededor de $-1^{\circ}$, comparado con los valores en eversión generados al momento de impactar $\left(-3.1 \pm 1.5^{\circ}\right)$; al respecto también detalla que el desplazamiento angular, al finalizar el impacto, es de 7 y $8^{\circ}$ y continúa aumentando cercano a los $10^{\circ}$. La fase de seguimiento representa el fin de la técnica y comienza cuando el deportista pierde contacto con el balón, por tanto, se podría clasificar como un movimiento inercial donde la viscoelasticidad muscular tiene una relevancia mayor, por tanto, el trabajo de estiramiento es importante, ya que permitiría el aumento del desplazamiento angular. En ese sentido, el trabajo de estiramiento dinámico presenta mejoras significativas para los rangos articulares. El modelo técnico de pateo en fútbol se presenta en la Tabla 2. 
MHSAlud, ISSN: 1659-097X, 17(2), Julio-Diciembre, 2020, PP 1-17

Aedo-Muñoz, Abarca-Reyes, Torres-Moreno, Bascuñan-Mosqueira, José Brito, Miarka, Caro-San Juan, Sánchez-Ramírez, Poblete-Gálvez

TABLA 2A.

Modelo técnico e indicadores biomecánicos por fase

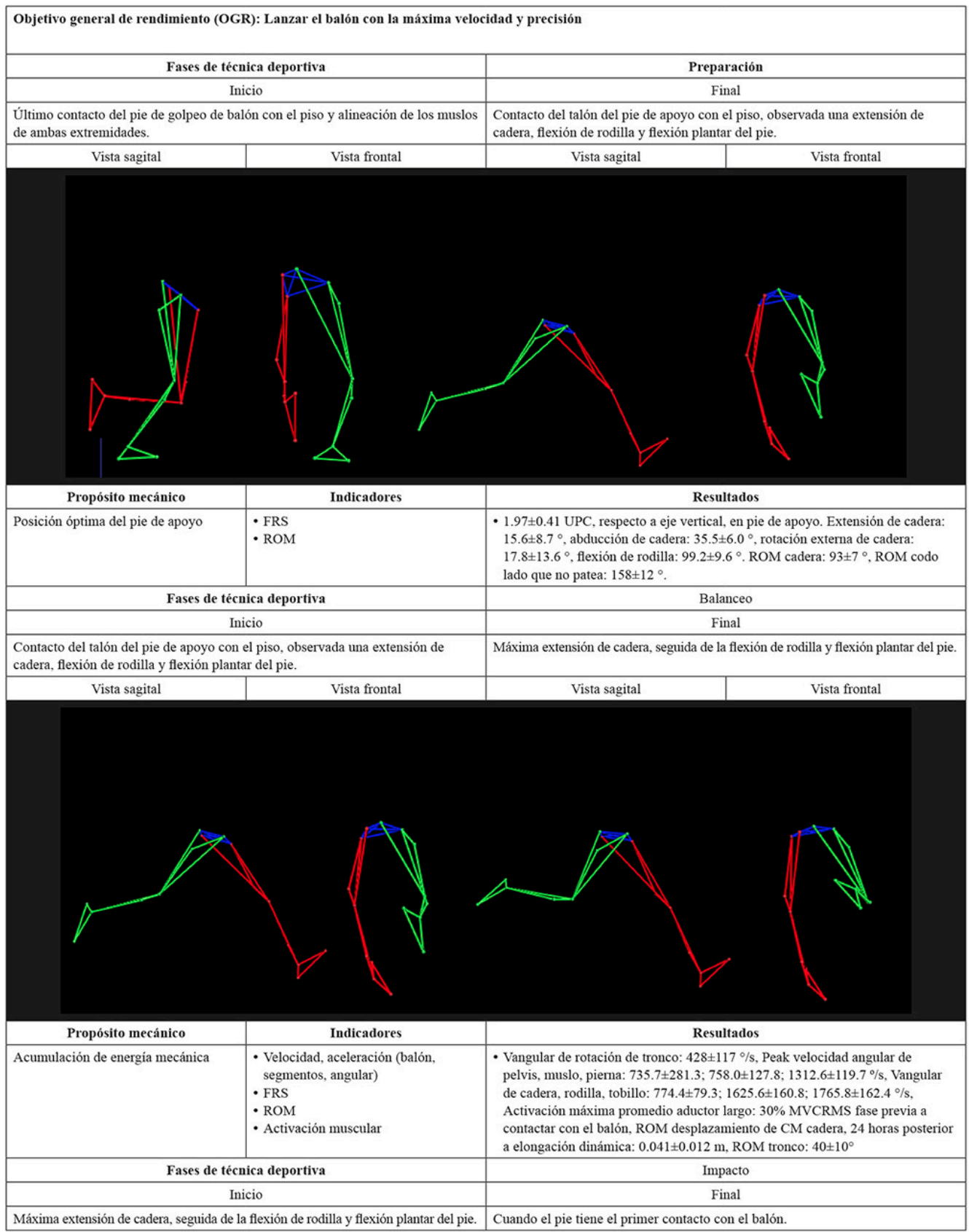


MHSALUd, ISSN: 1659-097X, 17(2), Julio-Diciembre, 2020, pP 1-17 Aedo-Muñoz, Abarca-Reyes, Torres-Moreno, Bascuñan-Mosqueira, José Brito, Miarka, Caro-San Juan, Sánchez-Ramírez, Poblete-Gálvez

\section{TABLA2B}

\section{Continuación}

Continuación

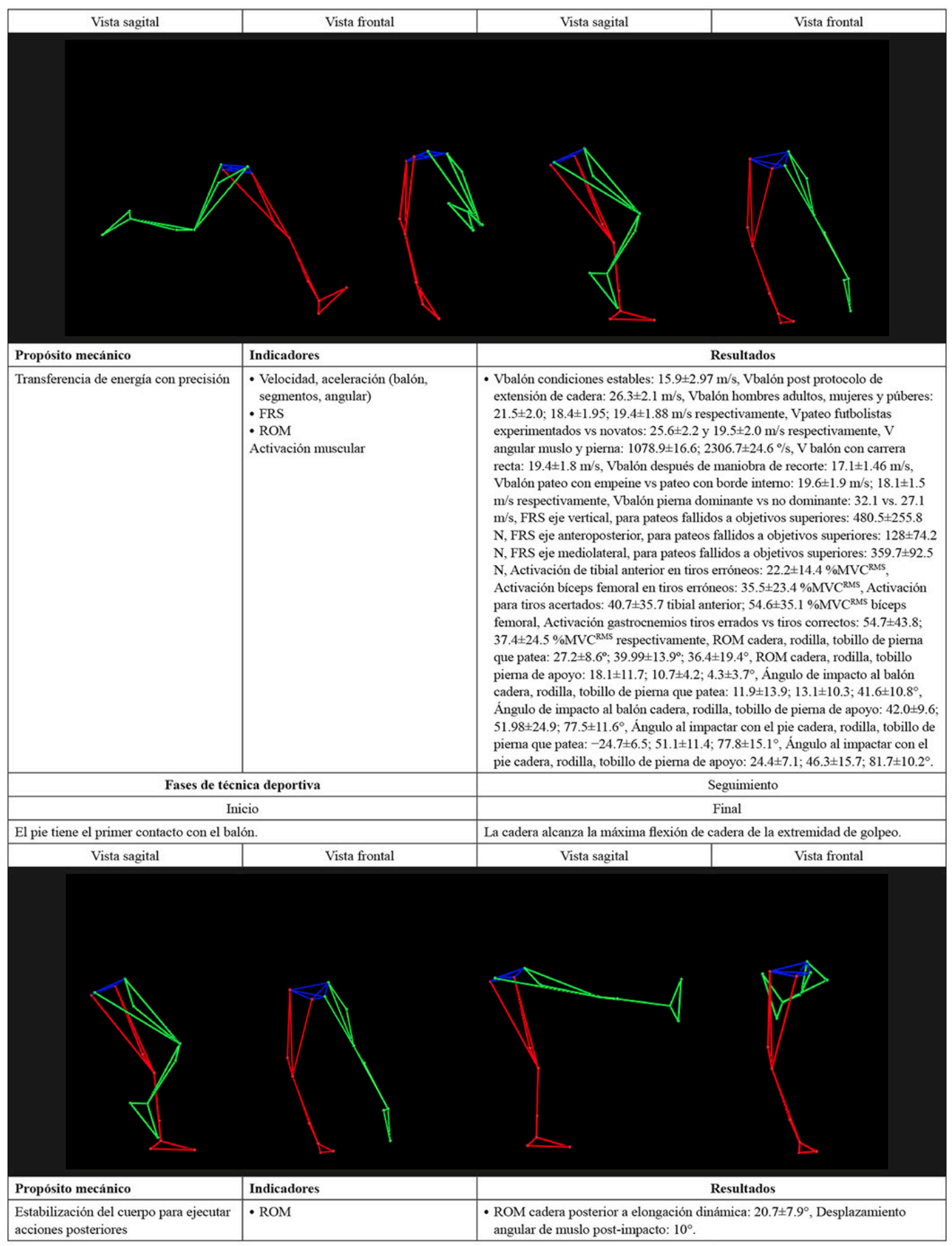

Nota: $\mathrm{V}=$ Velocidad $-\mathrm{UPC}=$ Unidad de peso corporal $-\mathrm{ROM}=$ Rango de movimiento FRS $=$ Fuerza de reacción del suelo $-\%$ MVCRMS $=\%$ Contracción voluntaria máxima $\mathrm{N}=$ Newton - Hemicuerpo verde $=$ Extremidad de golpeo - Hemicuerpo rojo $=$ Extremidad de apoyo. Elaboración propia de la investigación. 
MHSALUD, ISSN: 1659-097X, 17(2), Julio-Diciembre, 2020, PP 1-17

Aedo-Muñoz, Abarca-Reyes, Torres-Moreno, Bascuñan-Mosqueira, José Brito, Miarka, Caro-San Juan,

SÁnchez-Ramírez, Poblete-Gálvez

\section{Conclusiones}

Este artículo entregó una visión propositiva de la bibliografía actual sobre la técnica de pateo en fútbol. Identifica las variables cinéticas y cinemáticas de esta técnica, relacionándolas a través de las fases de la técnica y sus PM, para generar un modelo técnico de pateo en fútbol que responda, de manera precisa, al OGR de esta técnica.

Los principales resultados mostraron la relación que tienen estos estudios con las fases de la técnica. La mayor cantidad de artículos describen las fases desde cuando la pierna de pateo comienza a acelerar para impactar el balón, hasta el momento mismo de impacto de balón. Describe cuatro fases al respecto.

En la primera fase de preparación describió los principales IB. Éstos son FRS y ROM de cadera y rodilla. En la fase de balanceo e impacto los IB fueron la velocidad, ROM, FRS y activación muscular; mientras que en la fase de seguimiento el ROM resultó ser el principal indicador.

Estos IB son prioritarios para proporcionar un enfoque coherente en las distintas decisiones y situaciones que enfrentan los profesionales de la actividad física, la educación física, de entrenamiento y de ciencias de la salud en relación con la biomecánica y su aplicación en la técnica del pateo en fútbol. Esto, por cuanto propone un modelo técnico, con fuerte sustentación científica, y permite crear, alrededor de él, programas, protocolos y estrategias de enseñanza para el análisis de la técnica y su mejoramiento, como también programas que busquen el fortalecimiento de estos IB para la prevención de lesiones.

\section{ReFERENCIAS}

Augustus, S., Mundy, P. \& Smith, N. (2017). Support leg action can contribute to maximal instep soccer kick performance: an intervention study. Journal of Sports Sciences, 35(1), 89-98. https://doi.org/10.1080/026404 14.2016 .1156728

Amiri-Khorasani, M. Abu Osman, NA \& Yusof A. (2011). Acute effect of static and dynamic stretching on hip dynamic range of motion during instep kicking in professional soccer players. Journal of Strength and Conditioning Research, 25(6), 1647-1652. https://doi.org/10.1519/JSC.0b013e3181db9f41

Amiri-Khorasani, M. \& Kellis, E. (2013). Static vs. Dynamic Acute Stretching Effect on Quadriceps Muscle Activity during Soccer Instep Kicking. Journal of Human Kinetics, 39, 37-47. https://doi.org/10.2478/hukin-2013-00 66.

Amiri-Khorasani, M., Calleja-Gonzalez, J. \& Mogharabi-Manzari, M. (2016). Acute Effect of Different Combined Stretching Methods on Acceleration and Speed in Soccer Players. Journal of Human Kinetics, 50, 179-186. ht tp://doi.org/10.1515/hukin-2015-0154

Andersen, T. B. \& Dorge, H. C. (2011). The influence of speed of approach and accuracy constraint on the maximal speed of the ball in soccer kicking. Scandinavian Journal of Science \& Medicine in Sports, 21(1), 79-84. https:/ /doi.org/10.1111/j.1600-0838.2009.01024.x

Bangsbo, J. (1994). Energy demands in competitive soccer. Journal of Sports Sciences, 12 (Spec No), S5-12.

Bangsbo, J., Mohr M. \& Krustrup P. (2006) Physical and metabolic demands of training and match-play in the elite football player. Journal of Sports Sciences, 24(7), 665-674, https://doi.org/10.1080/02640410500482529

Brophy, RH., Backus, S. I., Pansy, B. S., Lyman, S. \& Williams, R. J. (2007). Lower Extremity Muscle Activation and Alignment During the Soccer Instep and Side-foot Kicks.Journal of Orthopedic \& Sports Physical Therapy, 37(5), 260-268. https://doi.org/10.2519/jospt.2007.2255

Charnock, BL., Lewis, C. L., Garrett, WEJ., \& Queen, R. M. (2009). Adductor longus mechanics during the maximal effort soccer kick. Sports Biomechanics, 8(3), 223-234. https://doi.org/10.1080/14763140903229500

De Witt, J. \& Hinrichs, R. (2012). Mechanical factor associated with the development of high ball velocity during an instep soccer kick. Sports Biomechanics, 11(3), 382-90. https://doi.org/10.1080/14763141.2012.661757 
MHSALUD, ISSN: 1659-097X, 17(2), JuLIO-DiCIEMBRE, 2020, PP 1-17 Aedo-Muñoz, Abarca-Reyes, Torres-Moreno, Bascuñan-Mosqueira, José Brito, Miarka, Caro-San Juan, Sánchez-Ramírez, Poblete-Gálvez

Diener-González, L. y Aedo-Muñoz, E. (2017). Indicadores cinemáticos del salto en extensión. Revista Arrancada, 17(30), 75-83. http://revistarrancada.cujae.edu.cu/index.php/arrancada/article/view/9-31/161

Dörge, H. C., Bull Anderson, T.; Sorensen H. \& Simonsen E. B. (2002). Biomechanical differences in soccer kicking with the preferred and the non-preferred leg. Journal of Sports Science, 20(4), 293-299. https://doi.org/10.10 $80 / 026404102753576062$

Egan, C. D., Verheul, M. H. G. \& Savelsbergh, G. J. P. (2010). Effects of experience on the coordination of internally and externally timed soccer kicks. Journal of Motor Behavior, 39(5), 423-432. https://doi.org/10.3200/JMBR 39.5.423-432

Fullenkamp, C.A.; Laurent, B. M., Matthew C. \& Amanda Paige, L. (2015). The contribution of trunk axial kinematics to post strike ball velocity during maximal instep soccer kicking. Journal of Applied Biomechanics, 31(5), 370-376. https://doi.org/10.1123/jab.2014-0188

García-Ruiz, S., Díaz-Hernández, T., Rozo-Lesmes, P., \& Espinosa de Gutiérrez, G. (2010). El enfoque poblacional\#: Las personas como centro de las políticas Population Focus: Persons as Focus of Policies. Revista de Investigaciones En Seguridad Social y Salud, 12, 129-142. http://uvsalud.univalle.edu.co/escuelas/rehabilitacio n/Logros/Publicaciones/523_11._el_enfoque_poblacional_las_personas\%5B1\%5D.pdf

González-Ródenas, J., Bondía, I. L., Moreno, F. C. y Malavés, R. A. (2015). Indicadores tácticos asociados a la creación de ocasiones de gol en fútbol professional. Cultura, Ciencia y Deporte, 10(30), 215-225. https://doi.org/10.12 800/ccd.v10i30.590

Inoue, K., Nunome, H., Sterzing, T., Shinkai, H \& Ikegami, Y. (2014). Dynamics of the support leg in soccer instep kicking. Journal of Sports Sciences, 32(11), 1023-1032. https://doi.org/10.1080/02640414.2014.886126

Ishii, H., Sakurai, Y., \& Maruyama, T. (2009). Effect of soccer shoe upper on ball behavior in curve kicks. Scientific Reports, 4, 60-67. http://dx.doi.org/10.1038/srep06067

Izquierdo, M., Echeverría, J. M. y Morante, J. C. (2008). Estructura y análisis del movimiento. En Biomecánica y bases neuromusculares de la actividad fisica y el deporte (pp. 770). Panamericana.

Jensen, J., Bandholm, T., Hölmich, P. \& Thorborg, K. (2014). Acute and sub-acute effects of repetitive kicking on hip adduction torque in injury-free elite youth soccer players. Journal of Sports Sciences, 32(14), 1357-1364. https: //doi.org/10.1080/02640414.2013.879673

Katis, A., Giannadakis, E., Kannas, T., Amiridis, I., Kellis, E., \& Lees, A. (2013). Mechanisms that influence accuracy of the soccer kick. Journal Electromyography and Kinesiology, 23(1), 125-131. https://doi.org/10.1016/j.jelek in.2012.08.020

Katis, A., Kellis, E., \& Lees, A. (2015). Age and gender differences in kinematics of powerful instep kicks in soccer. Sports Biomechanics, 14(3), 287 299. https://doi.org/10.1080/14763141.2015.1056221

Katis, A., \& Kellis, E. (2010). Three-dimensional kinematics and ground reaction forces during the instep and outstep soccer kicks in pubertal players. Journal of Sports Sciences, 28(11), 1233-1241. https://doi.org/10.1080/02640 414.2010.504781

Katis, A., \& Kellis, E. (2011). Is soccer kick performance better after a "faking" (cutting) maneuver task? Sports Biomechanics, 10(1), 35-45. https://doi.org/10.1080/14763141.2010.547594

Kawamoto, R., Miyagi, O., Ohashi, J., \& Fukashiro, S. (2007). Kinetic comparison of a side-foot soccer kick between experienced and inexperienced players. Sports Biomechanics, 6(2), 187-198. https://doi.org/10.1080/1476314 0701324966

Liberati, A., Altman, D. G., Tetzlaff, J., Mulrow, C., Gotzsche, P. C., Ioannidis, J. P. A., \& Moher, D. (2009). The PRISMA statement for reporting systematic reviews and meta-analyses of studies that evaluate health care interventions: explanation and elaboration. PLoS Medicine, 6(7), 1-28, e1000100. https://doi.org/https://doi .org/10.1371/journal.pmed.1000100

Mcmillan, K., Helgerud, J., Grant, S. J., Newell, J., Wilson, J., Macdonald, R., \& Hoff, J. (2005). Lactate threshold responses to a season of professional British youth soccer. British Journal of Sports Medicine, 39(7), 432-436. h ttps://doi.org/10.1136/bjsm.2004.012260 
MHSALUd, ISSN: 1659-097X, 17(2), Julio-Diciembre, 2020, PP 1-17

Aedo-Muñoz, Abarca-Reyes, Torres-Moreno, Bascuñan-Mosqueira, José Brito, Miarka, Caro-San Juan,

SÁnchez-Ramírez, Poblete-Gálvez

Moher, D., Liberati, A., Tetzlaff, J., \& Altman, D. G. (2009). Preferred reporting items for systematic reviews and metaanalyses: The PRISMA statement. PLoS Medicine, 6(7), 1-6. https://doi.org/10.1371/journal.pmed.1000097

Naito, K., Fukui, Y., \& Maruyama, T. (2010). Multijoint kinetic chain analysis of knee extension during the soccer instep kick. Human Movement Science, 29(2), 259-276. https://doi.org/10.1016/j.humov.2009.04.008

Navandar, A., Veiga, S., Torres, G., Chorro, D., \& Navarro, E. (2018). A previous hamstring injury affects kicking mechanics in soccer players. The Journal of Sports Medicine and Physical Fitness, 58(12), 1815-1822. https://d oi.org/10.23736/S0022-4707.18.07852-0

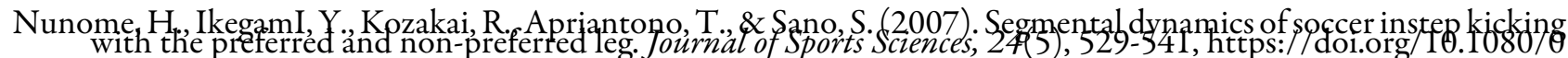
2640410500298024

Radman, I., Wessner, B., Bachl, N., Ruzic, L., Hackl, M., Prpic, T., \& Markovic, G. (2016). The acute effects of graded physiological strain on soccer kicking performance: a randomized, controlled cross-over study. European Journal of Applied Physiology, 116(2), 373-382. doi: https://doi.org/10.1007/s00421-015-3293-7

Orloff, H., Sumida, B., Chow, J., Habibi, L., Fujino, A \& Kramer, B. (2008). Ground reaction forces and kinematics of plant leg position during instep kicking in male and female collegiate soccer players. Sports Biomechanics, 7(2), 238-247. https://doi.org/10.1080/14763140701841704

Sasadai, J., Urabe, Y., Maeda, N., Shinohara, H., \& Fujii, E. (2015). The effect of ankle taping to restrict plantar flexion on ball and foot velocity during an instep kick in soccer. Journal of Sport Rehabilitation, 24(3), 261-267. https ://doi.org/10.1123/jsr.2014-0156

Sidaway, B., Bouchard, M., Chasse, J., Dunn, J., Govoni, A., McPherson, B., Roy, K., \& Anderson, D.I. (2017). The Role of Postural Support in Young Adults' Control of Stationary Kicking. Research Quarterly for Exercise and Sport, 88(2), 215-222. https://doi.org/10.1080/02701367.2017.1303134

Shan, G., \& Westerhoff, P. (2007). Fu11 - body kinematic characteristics of the maximal instep Soccer kick by male soccer players and parameters related to kick quality. Soccer, Sports Biomechanics, 4(1), 59-72. https://doi.org/ $10.1080 / 14763140508522852$

Shinkai H., Nunome H., Isokawa M., \& Ikegami. Y. (2009). Ball impact dynamics of instep soccer kicking. Medicine and Science in Sports and Exercise, 41(4), 889-97. https://doi.org/10.1249/MSS.0b013e31818e8044

Sinclair, J., Fewtrell, D., Taylor, P.J., Bottoms, L., Atkins, S., \& Hobbs, S. J. (2014). Three-dimensional kinematic correlates of ball velocity during maximal instep soccer kicking in males. European Journal of Sport Science, 14(8), 799-805. https://doi.org/10.1080/02640414.2014.965188

Sterzing T., \& Henning E. M. (2008). The influence of soccer shoes on kicking velocity in full-instep kicks. Exercise and Sport Science Review, 36(2), 91-97. https://doi.org/10.1097/JES.0b013e318168ece7

Stolen, T., Chamari, K., Castagna, C., \& Wisloff, U. (2005). Physiology of soccer: an update. Sports Medicine (Auckland, N.Z.), 35(6), 501-536. https://doi.org/10.2165/00007256-200535060-00004

Van den Tillaar, R., \& Ulvik, A. (2014). Influence of Instruction on Velocity and Accuracy in Soccer Kicking of Experienced Soccer Players. Journal of Motor Behavior, 46(5), 287-291. https://doi.org/10.1080/00222895.20 14.898609

Wells, G., Shea, B., O'Connell, D., Peterson, J., Welch, V., Losos, M., \& Tugwell, P. (2000). The Newcastle-Ottawa Scale (NOS) for Assessing the Quality of Non-Randomized Studies in Meta-Analysis. http://www.ohri.ca/progra $\mathrm{ms} /$ clinical_epidemiology/oxford.asp

\section{BY-NC-ND}

